\title{
Developing Optimum ANN Model for Mass Transfer with Chemical Reaction in Packed Column for Air-Carbon Dioxide and Aqueous Sodium Hydroxide System
}

\author{
S.L.Pandharipande \\ Associate Professor \\ Department of Chemical Engineering \\ Laxminarayan Institute of Technolgy, Nagpur
}

\author{
Ankit Singh \\ M.Tech $4^{\text {th }}$ sem \\ Department of Chemical Engineering \\ Laxminarayan Institute of Technolgy, Nagpur
}

\begin{abstract}
Gas-liquid reactions occupy a large share of the chemical reactions that are carried out industrially. The estimation of reaction rate is the most primary but complex task and is a prerequisite in design \& scaling up of reactors. Reaction rate is a function of number of parameters that include flow rates of gas and liquid phases, concentration of reactants and interfacial surface area, among others. Artificial Neural Network (ANN) is viewed as a black box modeling tool and has been applied for modeling of several chemical engineering operations. The present work is aimed at developing artificial neural network model for estimation of interfacial area as a function of gas \& liquid flow rates and inlet concentrations of gas \& liquid phases. Fifteen ANN models with different topologies have been developed and three models S-20, M-20 and C-50 are shortlisted based on better RMSE values. Further, model $\mathrm{C}-50$ has been observed to be highly acceptable based on comparison of relative error for all the data points.
\end{abstract}

\section{INTRODUCTION}

Gas-liquid reactions occupy a major share of the chemical reactions that are carried out industrially. Gas-liquid contactors are frequently employed in chemical process industry where these phases are brought into contact with each other and mass transfer between the gas and the liquid phases takes place. A good understanding of the behavior of gas-liquid contactors is essential for design purposes. Various types of reactions which can be defined by regimes include instantaneous reactions taking place at interface, fast reactions in liquid film, and slow reactions in bulk of liquid phase. The estimation of reaction rate is the most primary but complex task and is a prerequisite in design \& scaling up of reactors. As the reaction rate is a function of parameters like flow rates of gas and liquid phases, concentration of reactants and interfacial surface area, the estimation of these parameters are important.

An Artificial Neural Network (ANN) is a black box modeling tool that is based upon the working principle of the biological nervous system, such as brain processes information. [13], [18]. There are various types of ANN \& Multi Layer Perceptron (MLP) is one amongst them.It is a typical feed forward neural network that is applied in modeling of chemical processes \& consists of input, hidden \& output layers in that order. The nodes between the two successive layers are fully interconnected by means of constants called as weights. The outputs from the input layer nodes are fed to the nodes of the first hidden layer which in turn feed their outputs to the nodes of the next hidden layer. The hidden layer nodes process the input with the net activation function, such as the logistic sigmoid to compute their outputs. Training of the network is to be carried out \& there are few training algorithms reported in the literature. Rummelhart algorithm[29] is popular that is based on the Windro-Hoff rule known as Generalized Delta Rule (GDR).

Various applications of ANN in modeling, simulation and optimization of chemical processes have been reported in literature, these include, estimation of composition of ternary liquid mixture with its physical properties such as refractive index, $\mathrm{pH}$, conductivity [27], modeling for adsorption of dyes from aqueous solution using rice husk carbon [28], an approach to fault diagnosis in chemical processes [16], fault diagnosis in complex chemical plants [17], incipient fault diagnosis of chemical process [30], leak detection in liquefied gas pipeline [14], [19], for estimation of mass transfer coefficient for fast fluidized bed solids [31] , modeling of distillation column [15], detergent formulation [22], modeling of unsteady heat conduction in semi infinite solid [20], prediction of mass transfer coefficient in downflow jet loop reactor [21] and modeling of packed column [23] and similar other [24],[25] are also reported.

Researchers have reported estimation of interfacial area for various systems. This includes Kinetics of $\mathrm{CO} 2$ absorptionIII: First-order reaction in a packed column[1], Effective interfacial area in a packed column [2], Chemical reaction and effective interfacial areas in gas absorption [3], Effective interfacial area and liquid and gas side mass transfer coefficients in a packed column [4], New systems and methods for the measurement of effective interfacial area and mass transfer coefficients in gas-liquid contactors [5], and similar other [6],[7].[8] are also reported

\section{MATERIALS \& METHODS}

A typical reaction coming under the category of gas-liquid is air-carbon dioxide mixture contacted with sodium hydroxide solution is studied in the present work. It aims at estimation of interfacial area as a function of gas \& liquid flow rates, and inlet concentrations of gas \& liquid phases in a laboratory scale packed column. Various experimental runs are taken for measurement of parameters like outgoing concentrations of liquid \& gas phases for known parameters like flow rates and inlet concentrations of both the phases. The experimental data generated is used for calculation of effective interfacial area using theory of gas absorption accompanied by fast chemical reaction. 
The total data set consisting of 38 data points is divided in to two parts, training data set of 30 points and test data set of 8 points.

The details of ANN models developed in the present work using elite- $\mathbf{A N N ^ { \Theta }}{ }^{[26]}$ software with different topology are given in Table1. It is felt necessary to optimize the ANN topology with respect to iterations, number of hidden layers and number of neurons in each layer. Fifteen different ANN models having different combinations of topology parameters have been compared for their accuracy based on root mean square of error values. Best three of fifteen have been selected for further studies.

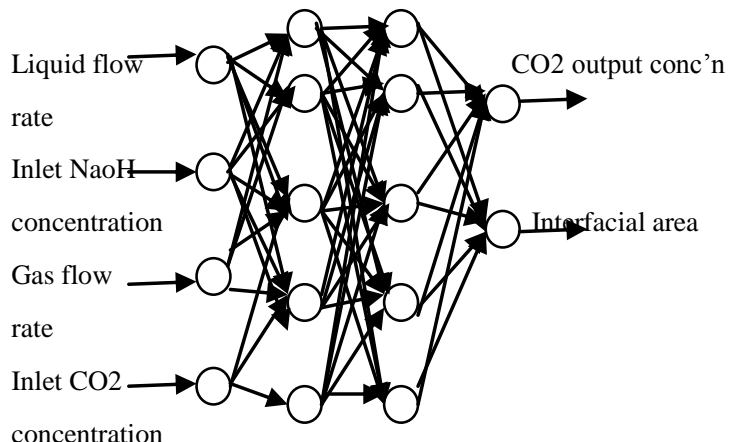

concentration

Fig 1: Typical topology for architecture of artificial neural network model used.

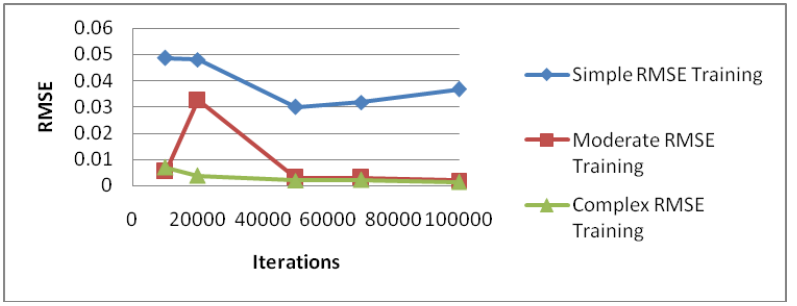

Fig 2: Comparison of RMSE of ANN models for training data set

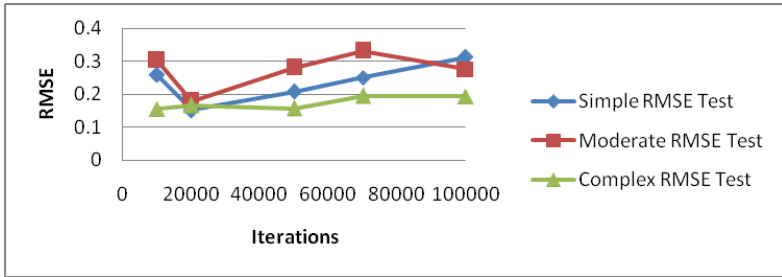

Fig 3: Comparison of RMSE of ANN models for test data set

Fig no $2 \& 3$ depict the RMSE of models based on simple, moderate and complex architectures for training and test data set respectively.

It can be seen from these figures that all the models developed using complex architecture have relatively lower values of RMSE for both training and test data sets. The interesting feature of the analysis is that the RMSE for ANN models based on moderate architecture has lower values for training data set than models based on simple architecture whereas RMSE trend is reversed for test data set values.
Based on the RMSE training and test data, models S-20, M-20 and C-50 are selected for further analysis due to their better accuracy of predictions. Comparison has been carried out between actual and predicted values of all the output parameters for the three ANN models shortlisted.

\section{RESULTS AND DISCUSSION:}

The graphs are plotted for the comparison of actual \& predicted values as shown in fig $4 \& 5$ for output parameters, outlet $\mathrm{CO}_{2}$ concentration and interfacial area for the training data set for ANN models S-20, M-20 and C-50.

Similarly graphs are also plotted for the comparison of actual \& predicted values as shown in fig $6 \& 7$ for output parameters, outlet $\mathrm{CO}_{2}$ concentration and interfacial area for both the test data set for ANN models S-20, M-20 and C-50.

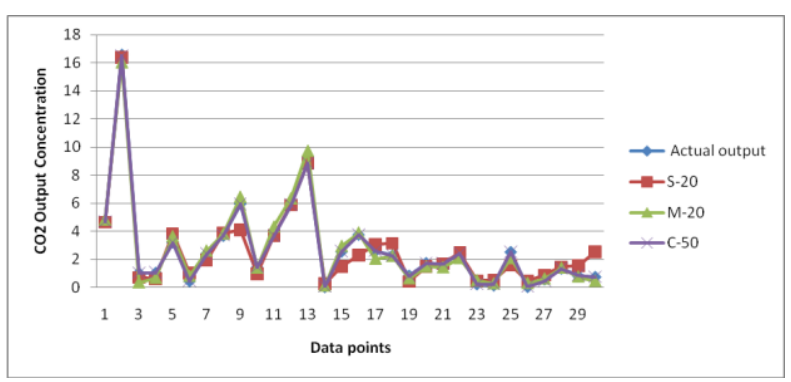

Fig 4: Comparison of all models for output $\mathrm{CO}_{2}$ concentration for training data set

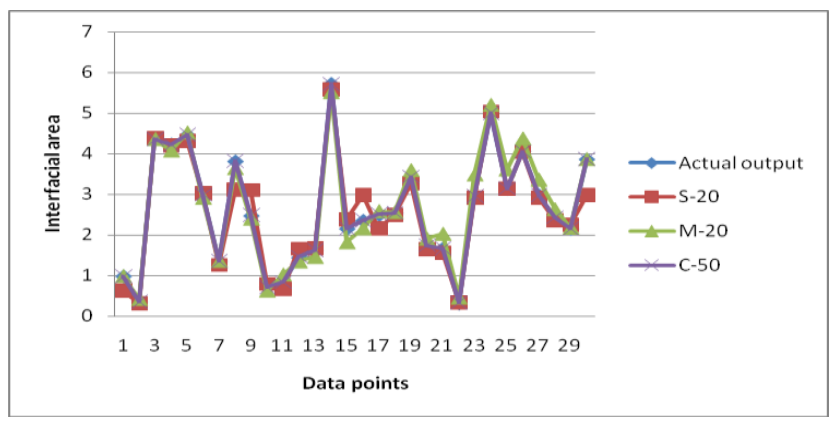

Fig 5: Comparison of all models for interfacial area for training data set

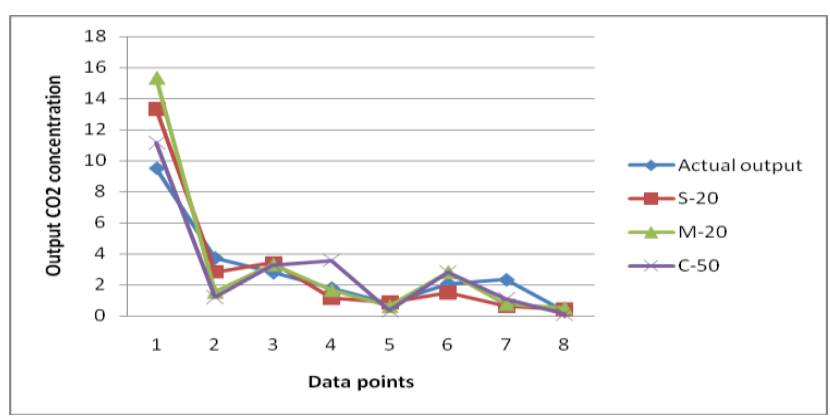

Fig 6: Comparison of all models for output $\mathrm{CO}_{2}$ concentration for test data set 


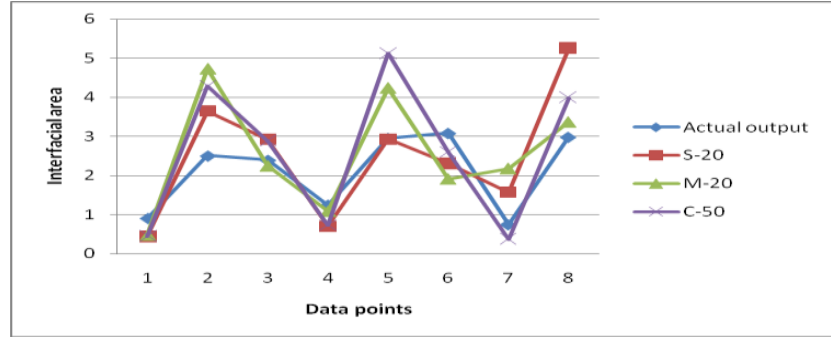

Fig 7: Comparison of all models for interfacial area for test data set

It can be said from these graphs that all the three ANN models have been fairly accurate in estimating the $\mathrm{CO} 2$ concentration and interfacial area for given set of operating conditions.

The criterion of selection of optimum ANN model amongst models S-20, M-20 and C-50 is based on the comparison of \% relative error values of all the data points. Table $2 \& 3$ highlight the range of the relative error for training \& test data set for these models whereas Fig 8, 9, 10, and 11 show the relative error for all the data points for output $\mathrm{CO}_{2}$ concentration and interfacial area respectively for training and test data set.

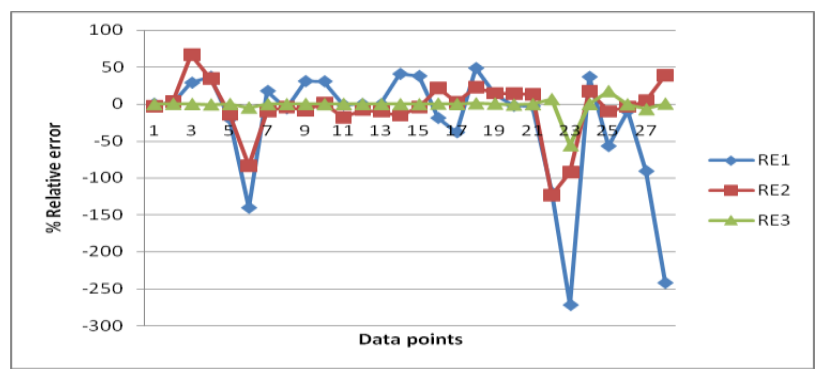

Fig 8: Comparison for relative error for output $\mathrm{CO}_{2}$ concentration for training data set

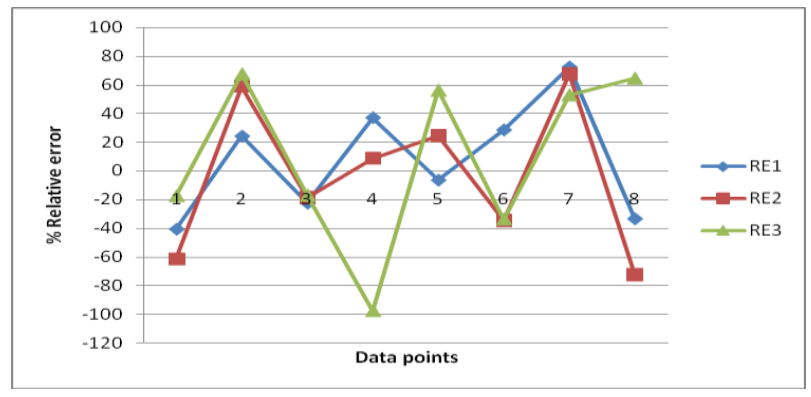

Fig 9: Comparison for relative error for output $\mathrm{CO}_{2}$ concentration for test data set

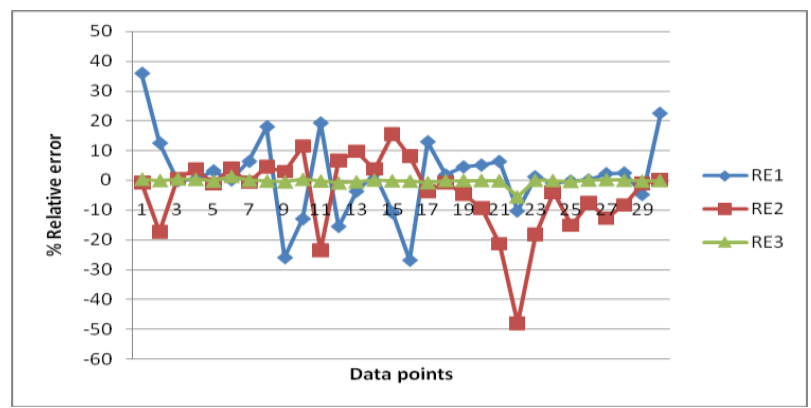

Fig 10: Comparison of relative error for interfacial area for training data set

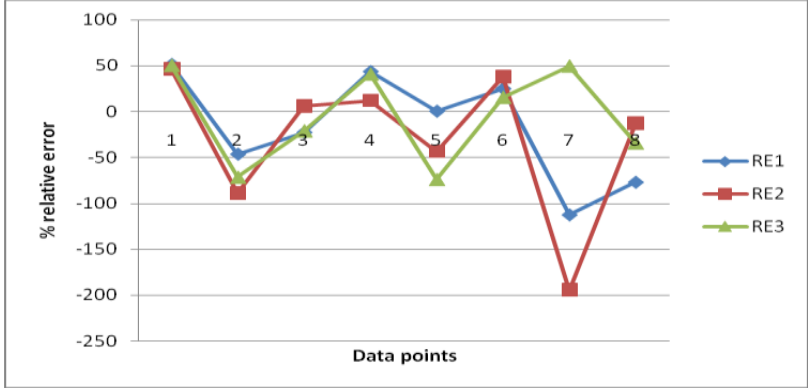

Fig 11: Comparison of relative error for interfacial area for test data set

\section{CONCLUSION:}

Gas-Liquid reactions occupy a major part of the chemical reactions carried out industrially. Chemical reactions have complex dynamics and involve multi variable non linear relationship while developing models related to them. Present work addressed to the estimation of interfacial area as a function of other parameters using artificial neural network and has been successful in developing models with high accuracy. The novel feature of this work is optimization of topology of ANN models to arrive at the more suitable model with better accuracy level for both the training and test data sets. Based on results and discussion it can be concluded that the ANN model having topology of three hidden layers with ten neurons each (C-50) has smaller relative error for all the data points which is satisfactory and acceptable.

\section{References}

[1] G.M. Richards, G.A. Ratcliff, P.V. Danckwerts, Chemical Engineering Science 19 (1964) 325.

[2] A.S. Jhaveri, M.M. Sharma, Chemical Engineering Science 23 (1968) 669.

[3] G.E.H. Joosten, P.V. Danckwerts, Chemical Engineering Science 28 (1973) 453.

[4] B.N. Sahay, M.M. Sharma, Chemical Engineering Science 28 (1973) 41.

[5] K. Sridharan, M.M. Sharma, Chemical Engineering Science 31 (1976) 767.

[6] V.A. Juvekar, M.M. Sharma, Chemical Engineering Science, 28 (1973) 976.

[7] M.H. Oyevaar, K.R. Westerterp, Chemical Engineering Science, 44 (1989) 2691.

[8] L. De Leye, G.F. Fromen, Computers and Chemical Engineering, 10 (1986) 493

[9] Nikolett Sipöcza, Finn Andrew Tobiesenb, Mohsen Assadia, Applied Energy 88 (2011) 2368.

[10] Jerzy Maćkowiak, Chemical Engineering Research and Design 89 (2011) 1308.

[11] H. Hikita, S. Asai, T. Takatsuka, The Chemical Engineering Journal 11 (1976) 131.

[12] Adisorn Aroonwilas, Amit Chakma, Paitoon Tontiwachwuthikul, Amornvadee Veawab, Chemical Engineering Science 58 (2003) 4037. 
[13] Anderson J. A. An Introduction to Neural Networks. New Delhi: Prentice-Hall of India, Pvt Ltd; 1999.

[14] Belsito S, Banerjee S. AIChEJ 44 (1998) 2675.

[15] Baratti R, Vacca G, Servida A. 74 (1995) 35.

[16] Fan J. Y, Nikolau M, White R. E. AIChEJ 39 (1993) 82.

[17] Hoskins J. C, Kaliyur K. M, Himmelblau D. M. AIChEJ, 37 (1991) 137.

[18] Pandharipande S.L. An Introduction to Artificial Neural Networks. Nagpur: Denett Publications; 2008.

[19] Pandharipande S. L, Badhe Y.P. Chem Eng World, 38 (2003) 70 .

[20] Pandharipande S.L, Badhe Y.P. Chem Eng World 38 (2003) 82 .

[21] Pandharipande S.L, Badhe Y.P. IChE, 45 (2003) 256.

[22] Pandharipande S. L, Agarwal R. S, Gogte B. B, Badhe Y. P. Chem Eng World, 38 (2003) 78.

[23] Pandharipande S. L, Mandavgane S. A. Indian J Chem Technol, 11 (2004) 820.
[24] Pandharipande S. L, Bhaise A., Poharkar A. Chem Eng world, 39 (2004) 50.

[25] Pandharipande S. L, Badhe Y. P. J Inst Eng, 84 (2004) 65.

[26] Pandharipande S. L, Badhe Y. P, 2004. elite-ANN@, ROC No SW-1471.

[27] Pandharipande S L, Shah A M, Heena T, International Journal of Computer Applications 41(2012) 23.

[28] Pandharipande S L, Khonde R D, International Journal of Computer Applications 41(2012)

[29] Rumelhart D. E, McClleland, Back Propagation Training Algorithm Processing, M.I.T Press, 1986 Cambridge Massachusetts.

[30] Watanabe K., Abe M., Kubota M., Himmelblau D. M. AIChEJ, 35 (1989) 137.

[31] Zamankhan P., Malinen P., Lepomaki H. AIChEJ, 43 (1997) 1684. 